\title{
Akt inhibitor MK-2206 reduces pancreatic cancer cell viability and increases the efficacy of gemcitabine
}

\author{
ZHANSHAN WANG ${ }^{1}$, GUANGTAO LUO ${ }^{2}$ and ZHENGJUN QIU ${ }^{1}$ \\ ${ }^{1}$ Department of General Surgery, Shanghai General Hospital of Nanjing Medical University, Shanghai 200080; \\ ${ }^{2}$ Department of General Surgery, The First Affiliated Hospital of Anhui Medical University, Hefei, Anhui 230022, P.R. China
}

Received January 27, 2019; Accepted November 15, 2019

DOI: $10.3892 / \mathrm{ol} .2020 .11300$

\begin{abstract}
The PI3K/Akt pathway is an attractive therapeutic target in the treatment of pancreatic cancer, as it was demonstrated to be aberrantly regulated in pancreatic cancer cells. The present study aimed to investigate the therapeutic potential of the novel Akt inhibitor MK-2206 in human pancreatic cancer cell lines. Pancreatic cancer cell survival following MK-2206 treatment was assessed using the Cell Counting Kit-8 (CCK-8) assay, colony formation and determination of the apoptotic rate by flow cytometry following annexin-V-fluorescein isothiocyanate/propidium iodide staining. The effects of MK-2206 alone or in combination with gemcitabine on pancreatic cell proliferation were assessed using the CCK- 8 assay. Western blotting was used to examine the effects of the two drugs on Akt protein expression. The results demonstrated that MK-2206 inhibited the proliferation and induced apoptosis of the $\mathrm{Mia} \mathrm{PaCa}-2$ and Panc-1 pancreatic cancer cell lines. In addition, CCK-8 cytotoxicity test showed that combined administration of MK-2206 with gemcitabine enhanced the cytotoxic efficacy of gemcitabine. Furthermore, a low dose of MK-2206 (1 $\mu \mathrm{M})$ combined with gemcitabine was enough to inhibit Akt phosphorylation. Taken together, these results provided some insight into the underlying mechanism of the anticancer effects of MK-2206 on pancreatic cancer cells.
\end{abstract}

\section{Introduction}

Pancreatic cancer is one of the most lethal human malignancies worldwide (1). To date, the causes underlying pancreatic cancer are still unknown, although certain risk factors have been identified. The possible risk factors for pancreatic cancer include sex (male), age ( $\geq 50)$, smoking, alcohol abuse, obesity, physical activities, diabetes, chronic

Correspondence to: Dr Zhengjun Qiu, Department of General Surgery, Shanghai General Hospital of Nanjing Medical University, 100 Haining Road, Shanghai 200080, P.R. China

E-mail: qiuzjdoctor@sina.com

Key words: Akt, MK-2206, pancreatic cancer, gemcitabine pancreatitis and genetic alterations (2). Patients diagnosed with pancreatic cancer typically have a poor prognosis $(1,2)$. Although mortality from the most common types of cancer has declined in the past few decades, the mortality of patients with pancreatic cancer remains high with a 5-year survival rate of only $6 \%$ (1). Surgical resection is the only potential curative therapy for pancreatic cancer. In the majority of cases, the disease has already metastasized to distant organs at the time of diagnosis $(1,2)$. Gemcitabine $(\mathrm{dFdC})$ is a deoxycytidine nucleoside analog, whose anti-proliferative properties are dependent on several inhibitory actions on DNA synthesis, blocking cell cycle progression at the $\mathrm{G}_{1} / \mathrm{S}$-phase boundary (3). Gemcitabine has been recognized as the standard first-line chemotherapy drug for pancreatic cancer. However, a previous study showed that gemcitabine can increase patient median survival by only 6 months (4). It is therefore crucial to develop novel therapeutic options and effective treatments for this disease.

It has been demonstrated that the PI3K/Akt pathway is excessively activated in various types of cancer, including pancreatic cancer (5). Furthermore, up to $60 \%$ of pancreatic cancer tissues and most pancreatic cancer cell lines exhibit increased Akt activity (6). Previous studies have reported that Akt is a major mediator of cell survival and apoptosis through the regulation of pro-survival and antiapoptotic proteins, including Bcl-XL and $\mathrm{NF}-\kappa \mathrm{B}$ in both normal and neoplastic cells $(7,8)$. Modulation of the Akt signaling pathway may therefore be considered a promising therapeutic approach for the treatment of pancreatic cancer.

MK-2206 is an allosteric Akt inhibitor that has been approved as an anti-cancer agent and that is administered orally in patients with cancer (such as renal cell carcinoma, parotid adenocarcinoma and colorectal cancer) (9). Previous studies have demonstrated that MK-2206 can suppress tumor growth (such as renal cell carcinoma, parotid adenocarcinoma and colorectal cancer) and enhance the antitumor efficacy of conventional chemotherapeutic agents, including docetaxel and carboplatin $(10,11)$. However, to the best of our knowledge, only a few studies $(10,11)$ have investigated the effect of MK-2206 on pancreatic cancer. The present study aimed to evaluate the antitumor effect of MK-2206 in pancreatic cancer cell lines in order to determine whether it may be considered as a promising therapeutic agent for the treatment of patients with pancreatic cancer. 


\section{Materials and methods}

Cell culture. The human pancreatic cancer lines Mia PaCa-2, Panc-1, BxPC-3, AsPC-1, Capan-2, CFPAC-1 and SW1990 were obtained from the American Type Culture Collection. All cell lines (Mia PaCa-2, Panc-1, Capan-2, CFPAC-1 and SW1990) were routinely maintained in Dulbecco's modified Eagle's medium (HyClone; GE Healthcare Life Sciences) or RPMI-1640 medium (HyClone; GE Healthcare Life Sciences) (BxPC-3 and AsPC-1) containing 10\% FBS (Gibco; Thermo Fisher Scientific, Inc.) in a humidified atmosphere of $5 \% \mathrm{CO}_{2}$ at $37^{\circ} \mathrm{C}$.

Reagents and antibodies. MK-2206 and gemcitabine were purchased from Selleck Chemicals. MK-2206 was dissolved in DMSO according to the instruction. Gemcitabine was dissolved in water according to the instruction. The primary antibodies against phosphorylated (p)-Akt (ser473) (cat. no. 3787), total (T)-Akt (cat. no. 4691) and $\beta$-actin (cat. no. 12262) were purchased from Cell Signaling Technology, Inc. Anti-rabbit IgG, HRP-linked secondary antibody (cat. no. 7074) were purchased from Cell Signaling Technology, Inc.

Western blotting. Pancreatic cells were lysed with 2X SDS lysis buffer. Bicinchoninic acid (EpiZyme) was used to quantify proteins. The amount of proteins loaded per lane was $20 \mu \mathrm{g}$. Proteins were separated by SDS-PAGE (separating gel concentration, 10\%; stacking gel concentration, 10\%) and transferred onto nitrocellulose membranes (Axygen; Corning, Inc.). Membranes were blocked with 5\% milk in Tris-buffered saline (TBS) for $1 \mathrm{~h}$ at room temperature, and incubated overnight with the primary antibodies (p-Akt $(1: 1,000)$, T-Akt $(1: 1,000)$ and $\beta$-actin $(1: 1,000)$ at $4^{\circ} \mathrm{C}$. After incubation with HRP-conjugated secondary antibodies (anti-rabbit secondary antibody for p-Akt and T-Akt, 1:5,000) for $1 \mathrm{~h}$ at room temperature, the membranes were washed 3 times with TBS for $10 \mathrm{~min}$. Bands were detected using enhanced chemiluminescence detection kit (EMD Millipore).

Cell viability assay. Mia PaCa- 2 and Panc- 1 cells were seeded in 96-well plates at a density of $5 \times 10^{3}$ cells/well. After $24 \mathrm{~h}$, the medium was replaced by various concentrations of drugs, and the cells were incubated for another 48 h. Cell viability was determined using Cell Counting Kit-8 (CCK-8; Dojindo Molecular Technologies, Inc.). The amount of CCK-8 reagent added per well was $10 \mu$ l. Cells were cultured in a humidified atmosphere of $5 \% \mathrm{CO}_{2}$ and $95 \%$ air at $37^{\circ} \mathrm{C}$. Absorbance was measured at $450 \mathrm{~nm}$ using a microplate reader.

Colony-formation assay. To assess the effect of MK-2206 on the colony formation of Mia PaCa-2 and Panc- 1 cell lines, cells were seeded in $3.5 \mathrm{~cm}$ dishes at a density of $1 \times 10^{3}$ cells/dish. The medium was renewed every three days until visible colonies were formed for one week. Cells were fixed with anhydrous methanol for $15 \mathrm{~min}$ and stained with $1 \%$ crystal violet for $10 \mathrm{~min}$ at room temperature. Colonies were captured with a camera.
Apoptosis detection. The apoptotic rates of $\mathrm{Mia} \mathrm{PaCa}-2$ and Panc-1 cell lines following treatment with MK-2206 were assessed by flow cytometry with Annexin-V/propidium iodide (PI) staining according to the manufacturer's instructions (cat. no. FA101-01; TransGen Biotech). Apoptotic rates were subsequently determined using a flow cytometer (LSRFortessa X-20; BD Biosciences).

Statistical analysis. All data were presented as the means \pm standard deviation. Three independent repeats were conducted for each experiment. Comparison between the groups was calculated using a two-tailed Student's t-test. $\mathrm{P}<0.05$ was considered to indicate a statistically significant difference.

\section{Results}

MK-2206 inhibits Akt activation in pancreatic cancer cell lines. The expression of p-Akt was examined in human pancreatic cancer cell lines. The results demonstrated that different levels of Akt were activated in different pancreatic caner cell lines (Fig. 1A). The effect of MK-2206 on Akt phosphorylation was subsequently examined. Treatment with MK-2206 was performed at 0.1 and $1 \mu \mathrm{M}$ for $48 \mathrm{~h}$. As presented in Fig. 1B, MK-2206 treatment reduced the expression p-Akt in all pancreatic cancer cell lines. No changes in the levels of total Akt protein were observed. These results demonstrated that MK-2206 inhibited Akt phosphorylation in pancreatic cancer cells.

MK-2206 inhibits the proliferation and induces the apoptosis of pancreatic cancer cells. CCK-8 assay was performed to examine the effects of MK-2206 on Mia PaCa-2 and Panc-1 cell proliferation. As presented in Fig. 2A, MK-2206 inhibited cell proliferation of human cancer cell lines. Furthermore, colony formation assay was performed to assess the effects of MK-2206 on the viability of Mia PaCa-2 and Panc-1 cells. The results demonstrated that the number of colonies formed following treatment with MK-2206 was reduced in both cell lines (Fig. 2B).

To assess MK-2206-induced apoptosis, Mia PaCa-2 and Panc- 1 cells were treated with DMSO $(0.1 \%)$ or $1 \mu \mathrm{M}$ MK-2206 for $48 \mathrm{~h}$, and the percentage of annexin V-PI positive cells was determined. The results demonstrated that treatment with MK-2206 for $48 \mathrm{~h}$ significantly induced apoptosis in the two pancreatic cancer cell lines (Fig. 2C and D).

MK-2206 increases the cytotoxic effects of gemcitabine in human pancreatic cancer cells. Gemcitabine has been extensively used in the treatment of pancreatic cancer (3). As presented in Fig. 3A and B, MK-2206 and gemcitabine co-treatment for $48 \mathrm{~h}$ significantly decreased cell proliferation compared with the gemcitabine and DMSO group. These data suggested that MK-2206 may enhance the cytotoxicity induced by gemcitabine in pancreatic cancer cells.

MK-2206 inhibits gemcitabine-induced Akt activation in pancreatic cancer cell lines. A previous study demonstrated that gemcitabine induces Akt activation in pancreatic cancer cells (12). Activation of the Akt survival pathway may therefore block the therapeutic efficacy of 
A

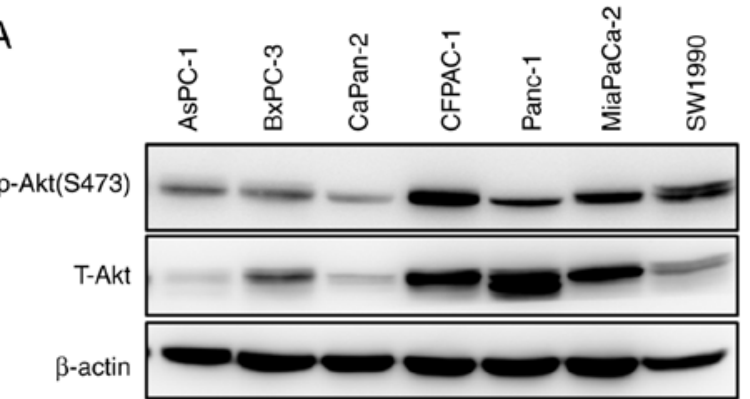

B
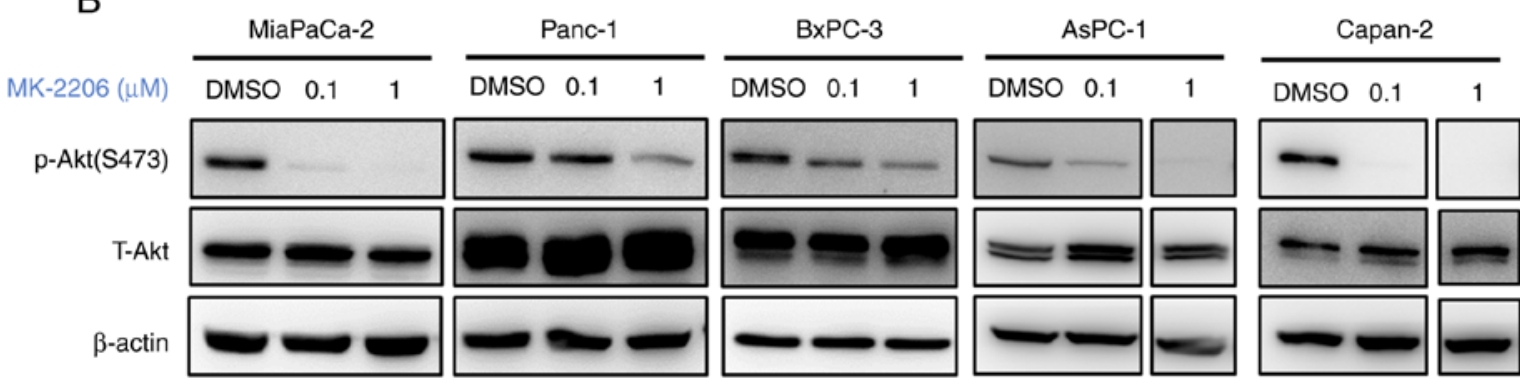

Figure 1. MK-2206 inhibits Akt phosphorylation in pancreatic cancer cell lines. (A) Expression of p-Akt and T-Akt was analyzed by western blotting in human pancreatic cancer cell lines. (B) Mia PaCa-2, Panc-1, BxPC-3, AsPC-1 and Capan-2 cells were treated with MK-2206 (0.1 and $1 \mu \mathrm{M})$ for $48 \mathrm{~h}$, and the expression of p-Akt and T-Akt was measured by western blotting. $\beta$-actin was used as a loading control. $n=3$. p, phosphorylated; $\mathrm{T}$, total.
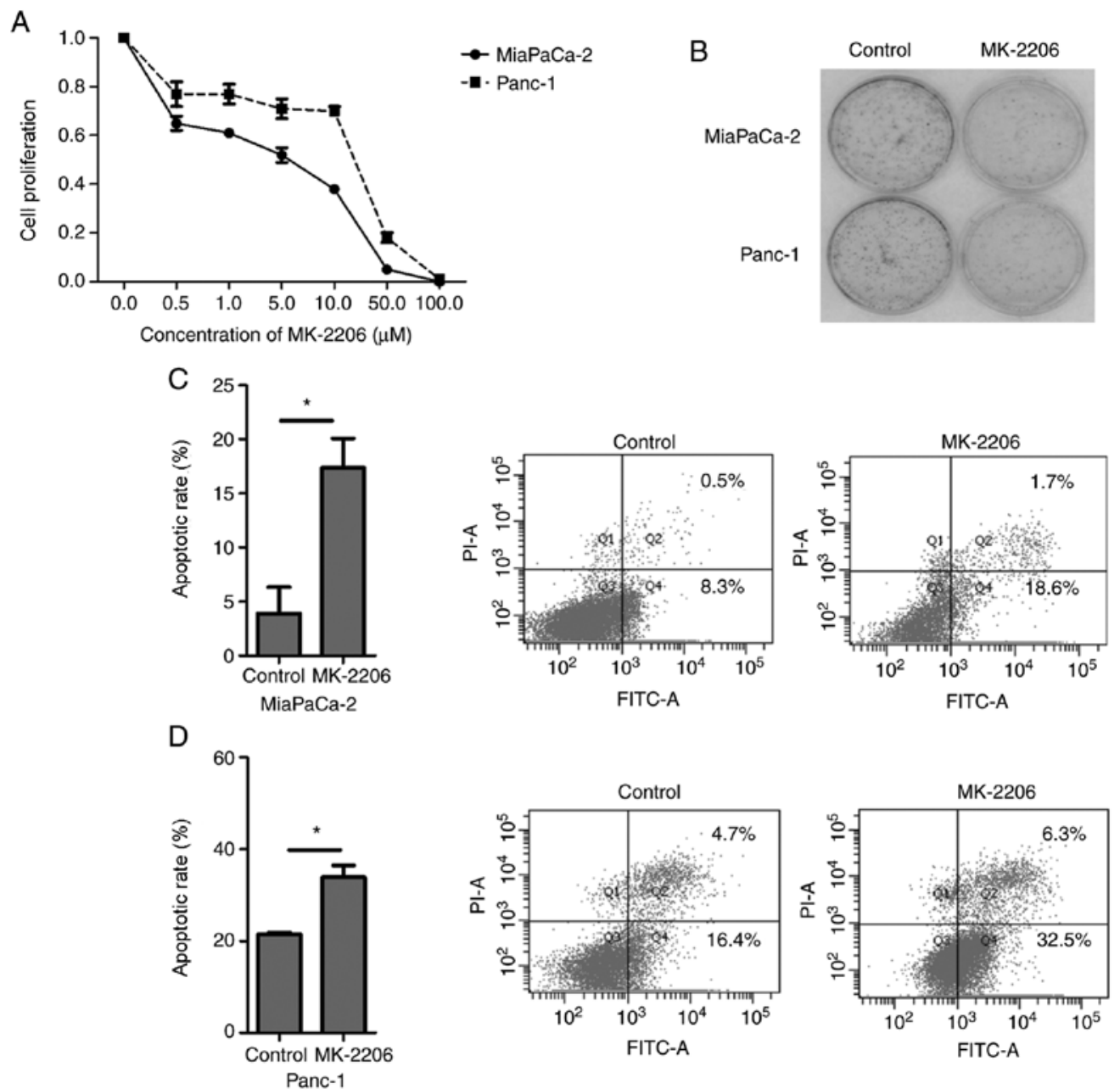

Figure 2. MK-2206 inhibits the proliferation and induces the apoptosis of pancreatic cancer cells. (A) Mia PaCa-2 and Panc-1 cells were treated with MK-2206 at the indicated concentration for $48 \mathrm{~h}$. Cell proliferation was evaluated using the Cell Counting Ki- 8 assay. (B) Colony formation was quantified 10 days post-treatment with MK-2206. (C) Mia PaCa-2 and (D) Panc-1 cells were treated with $1 \mu \mathrm{M}$ MK-2206 for $48 \mathrm{~h}$ prior to assessing the apoptotic rate by flow cytometry using annexin-V/PI staining. $n=3$. PI, propidium iodide. 
A

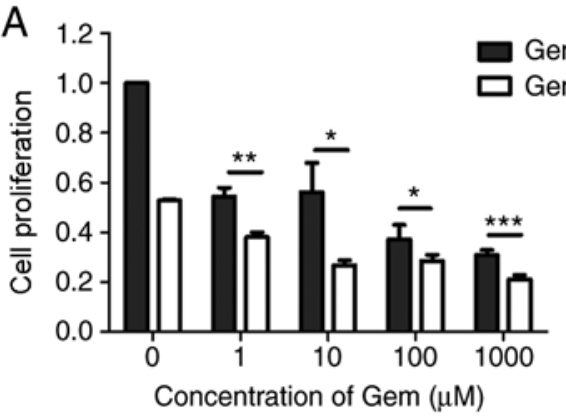

MiaPaCa-2

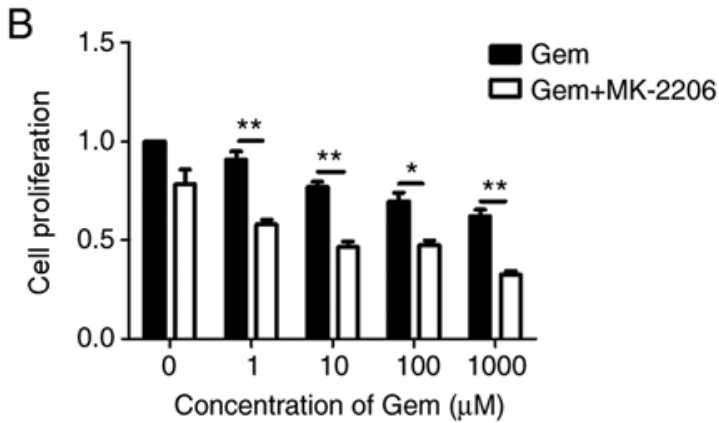

Panc-1

Figure 3. Co-treatment with MK-2206 and Gem increases the cytotoxic effect of gemcitabine. (A) Mia PaCa-2 and (B) Panc-1 cell lines were simultaneously treated with $1 \mu \mathrm{M}$ MK-2206 and Gem at the indicated concentration for $48 \mathrm{~h}$. Cell proliferation was evaluated using the Cell Counting kit 8 assay. $\mathrm{n}=3$. ${ }^{*} \mathrm{P}<0.05$, ${ }^{* *} \mathrm{P}<0.01$ and ${ }^{* * *} \mathrm{P}<0.005$ vs. gemcitabine treatment group. Gem, gemcitabine.
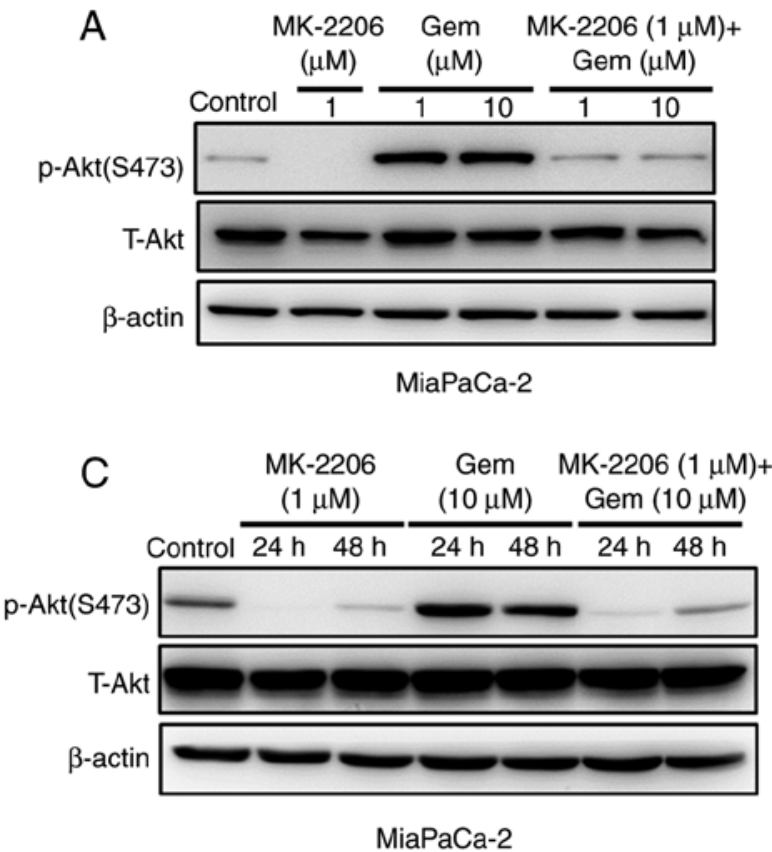
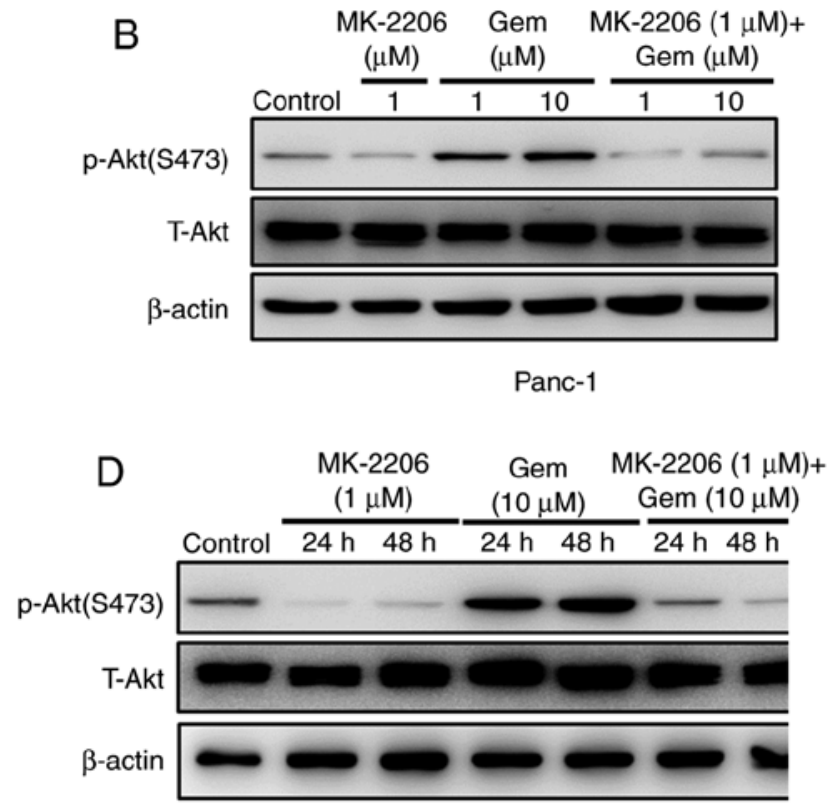

Panc-1

Figure 4. Co-treatment with MK-2206 and Gem inhibits the gemcitabine-induced Akt activation. (A and B) Cells were treated with Gem (1 or $10 \mu \mathrm{M})$ in the presence of MK-2206 (1 $\mu \mathrm{M})$ for $48 \mathrm{~h}$. MK-2206 inhibited Akt phosphorylation induced by Gem in (A) Mia PaCa-2 and (B) Panc-1 cell lines. (C and D) Time-dependent effects of MK-2206 and Gem on p-Akt and T-Akt expression in (C) Mia PaCa-2 and (D) Panc-1 cell lines. Cells were treated with $1 \mu \mathrm{M}$ MK-2206 alone, $10 \mu \mathrm{M}$ Gem alone or both agents for 24 and $48 \mathrm{~h}$. The expression levels of p-Akt and T-Akt were determined by western blotting. $\beta$-actin was used as a loading control. $\mathrm{n}=3$. Gem, gemcitabine; $\mathrm{p}$, phosphorylated; $\mathrm{T}$, total.

gemcitabine (10). To investigate whether MK-2206 may inhibit gemcitabine-induced Akt activation in pancreatic cancer cells, p-Akt expression in Mia PaCa-2 and Panc-1 cell lines was assessed following treatment with gemcitabine alone (1 or $10 \mu \mathrm{M}$ ) or in combination with $1 \mu \mathrm{M}$ MK-2206 for 24 and $48 \mathrm{~h}$. The results demonstrated that the expression of p-Akt (Ser473) was increased after treatment with 1 and $10 \mu \mathrm{M}$ gemcitabine for $48 \mathrm{~h}$ in Mia PaCa-2 and Panc-1 cell lines. However, gemcitabine-induced Akt phosphorylation was inhibited by $1 \mu \mathrm{M}$ MK-2206 in these two pancreatic cancer cell lines (Fig. 4A and B). In addition, $10 \mu \mathrm{M}$ gemcitabine treatment for 24 and $48 \mathrm{~h}$ increased Akt phosphorylation in Mia PaCa-2 and Panc-1 cell lines, and gemcitabine-induced Akt phosphorylation was inhibited by $1 \mu \mathrm{M}$ MK-2206 treatment for 24 and $48 \mathrm{~h}$ in these two pancreatic cancer cell lines (Fig. 4C and D).

\section{Discussion}

MK-2206 is a highly selective allosteric inhibitor of Akt that binds to the pleckstrin-homology domain of Akt, inducing a conformational change that prevents Akt localization to the plasma membrane and its subsequent activation (13). In the present study, human pancreatic cancer cell lines Mia PaCa-2, Panc-1, CFPAC-1 and SW1990 exhibited higher p-Akt expression compared with BxPC-3, AsPC-1 and Capan-2 cell lines. In addition, a previous study from our laboratory demonstrated that Mia PaCa- 2 and Panc- 1 cell lines were more resistant to gemcitabine compared with CFPAC-1 and SW1990 cell lines (data not shown). Thus, Mia PaCa-2 and Panc-1 cell lines were selected for subsequent experiments in the present study. The results of the present study demonstrated that MK-2206 decreased p-Akt expres- 
sion and reduced cell proliferation and colony formation in Mia PaCa-2 and Panc-1 cell lines. In addition, MK-2206 stimulated apoptosis in Mia PaCa-2 and Panc-1 cells. These results were consistent with previous studies on other types of cancer, including gastric cancer, neuroblastoma and thyroid cancer (14-16).

It is worth noting that the inhibition rate of MK-2206 in pancreatic cancer cells in Fig. 3A and B disagree with those of Fig. 2. This may have occurred due to the long-term use of the cell lines, as cellular viability decreases and cell senescence level increases with the extension of the passage number.

Gemcitabine, used alone or in combination with other therapeutic agents, is the first-line chemotherapy strategy for the treatment of locally advanced or metastatic pancreatic cancer $(4,17)$. However, most patients do not respond well and develop chemoresistance to gemcitabine (18). It has been reported that gemcitabine can activate the Akt signaling pathway in pancreatic cancer cells via the overproduction of reactive oxygen species, which reduces the anti-tumor responses and inhibits the therapeutic efficacy of gemcitabine (12). In the present study, MK-2206 treatment in combination with gemcitabine abolished the gemcitabine-induced Akt activation and increased the cytotoxic effect of gemcitabine. These findings were consistent with a previous study reporting that MK-2206 can sensitize human cancer cells to numerous chemotherapeutic agents, including doxorubicin and fluorouracil (10). Previous clinical phase 1 trial showed that MK-2206 combination with carboplatin and paclitaxel, docetaxel, or erlotinib, was well-tolerated at doses that inhibit Akt signaling (19). Furthermore, a number of ongoing or completed phase 2 trials have demonstrated that numerous tumor types are responsive to MK-2206 alone or in combination with other drugs $(20,21)$. Combining gemcitabine with MK-2206 may therefore be considered as a promising therapeutic strategy for the treatment of patients with pancreatic cancer.

In conclusion, the results of the present study demonstrated that MK-2206 inhibited Akt phosphorylation and attenuated pancreatic cancer cell proliferation. In addition, the combination of MK-2206 with gemcitabine enhanced pancreatic cancer cell proliferation inhibition, which may have been due to the MK-2206-mediated inhibition of gemcitabine-induced Akt activation. It is worth noting that the toxicity of MK-2206 on normal human pancreatic ductal epithelium cells was not examined in the present study. A previous study reported that MK-2206 was well tolerated in patients and that its toxic and side effects were acceptable (9). The combination of MK-2206 with gemcitabine may therefore help overcome chemoresistance in pancreatic cancer. Further in vivo investigation using patient-derived xenograft models is required to confirm these findings.

\section{Acknowledgements}

Not applicable.

\section{Funding}

This work was supported by the National Natural Science Foundation of China (grant no. 81372640) and the Shanghai Sailing Program (grant no. 17YF1415700).

\section{Availability of data and materials}

The datasets used and/or analyzed during the current study are available from the corresponding author on reasonable request.

\section{Authors' contributions}

ZW and GL conceived and designed the experiments. GL interpreted the data, performed the statistical analysis and analyzed the results. ZW and GL wrote the manuscript. ZW and $Z Q$ revised the manuscript. ZQ made substantial contributions to conception and design of the study. All authors read and approved the final version of the manuscript and agreed to be accountable for all aspects of the research in ensuring that the accuracy of integrity of any part of the work are appropriately investigated and resolved.

\section{Ethics approval and consent to participate}

Not applicable.

\section{Patient consent for publication}

Not applicable.

\section{Competing interests}

The authors declare that they have no competing interests.

\section{References}

1. Kamisawa T, Wood LD, Itoi T and Takaori K: Pancreatic cancer. Lancet 388: 73-85, 2016.

2. Zhang Q,Zeng L, Chen Y,Lian G, Qian C, Chen S,Li J and Huang K: Pancreatic cancer epidemiology, detection, and management. Gastroenterol Res Pract 2016, 8962321, 2016.

3. Zeng S, Pöttler M, Lan B, Grützmann R, Pilarsky C and Yang H: Chemoresistance in pancreatic cancer. Int J Mol Sci 20: E4504, 2019.

4. Oettle H, Post S, Neuhaus P, Gellert K, Langrehr J, Ridwelski K, Schramm H, Fahlke J, Zuelke C, Burkart C, et al: Adjuvant chemotherapy with gemcitabine vs observation in patients undergoing curative-intent resection of pancreatic cancer: A randomized controlled trial. JAMA 297: 267-277, 2007.

5. Manning BD and Toker A: AKT/PKB signaling: Navigating the network. Cell 169: 381-405, 2017.

6. Arlt A, Müerköster SS and Schäfer H: Targeting apoptosis pathways in pancreatic cancer. Cancer Lett 332: 346-358, 2013.

7. Engelman JA, Luo J and Cantley LC: The evolution of phosphatidylinositol 3-kinases as regulators of growth and metabolism. Nat Rev Genet 7: 606-619, 2006.

8. Zhang XJ and Jia SS: Fisetin inhibits laryngeal carcinoma through regulation of $\mathrm{AKT} / \mathrm{NF}-\mathrm{\kappa B} / \mathrm{mTOR}$ and ERK1/2 signaling pathways. Biomed Pharmacother 83: 1164-1174, 2016.

9. Yap TA, Yan L, Patnaik A, Fearen I, Olmos D, Papadopoulos K, Baird RD, Delgado L, Taylor A, Lupinacci L, et al: First-in-man clinical trial of the oral pan-AKT inhibitor MK-2206 in patients with advanced solid tumors. J Clin Oncol 29: 4688-4701, 2011.

10. Hirai H, Sootome H, Nakatsuru Y, Miyama K, Taguchi S, Tsujioka K, Ueno Y, Hatch H, Majumder PK, Pan BS and Kotani H: MK-2206, an allosteric Akt inhibitor, enhances antitumor efficacy by standard chemotherapeutic agents or molecular targeted drugs in vitro and in vivo. Mol Cancer Ther 9: 1956-1967, 2010.

11. Lin YH, Chen BYH, Lai WT, Wu SF, Guh JH, Cheng AL and Hsu LC: The Akt inhibitor MK-2206 enhances the cytotoxicity of paclitaxel (Taxol) and cisplatin in ovarian cancer cells. Naunyn Schmiedebergs Arch Pharmacol 388: 19-31, 2015. 
12. Arora S, Bhardwaj A, Singh S, Srivastava SK, McClellan S, Nirodi CS, Piazza GA, Grizzle WE, Owen LB and Singh AP: An undesired effect of chemotherapy: Gemcitabine promotes pancreatic cancer cell invasiveness through reactive oxygen species-dependent, nuclear factor $\kappa \mathrm{B}$ - and hypoxia-inducible factor $1 \alpha$-mediated up-regulation of CXCR4. J Biol Chem 288: 21197-21207, 2013.

13. Okuzumi T, Fiedler D, Zhang C, Gray DC, Aizenstein B, Hoffman R and Shokat KM: Inhibitor hijacking of Akt activation. Nat Chem Biol 5: 484-493, 2009.

14. Jin P, Wong CC, Mei S, He X, Qian Y and Sun L: MK-2206 co-treatment with 5-fluorouracil or doxorubicin enhances chemosensitivity and apoptosis in gastric cancer by attenuation of Akt phosphorylation. OncoTargets Ther 9: 4387-4396, 2016.

15. Li Z, Yan S, Attayan N, Ramalingam S and Thiele CJ: Combination of an allosteric Akt Inhibitor MK-2206 with etoposide or rapamycin enhances the antitumor growth effect in neuroblastoma. Clin Cancer Res 18: 3603-3615, 2012.

16. Burke JF, Schlosser L, Harrison AD, Kunnimalaiyaan M and Chen H: MK-2206 causes growth suppression and reduces neuroendocrine tumor marker production in medullary thyroid cancer through Akt inhibition. Ann Surg Oncol 20: 3862-3868, 2013.
17. Burris HA III, Moore MJ, Andersen J, Green MR, Rothenberg ML, Modiano MR, Cripps MC, Portenoy RK, Storniolo AM, Tarassoff $\mathrm{P}$, et al: Improvements in survival and clinical benefit with gemcitabine as first-line therapy for patients with advanced pancreas cancer: A randomized trial. J Clin Oncol 15: 2403-2413, 1997.

18. Amrutkar M and Gladhaug IP: Pancreatic cancer chemoresistance to gemcitabine. Cancers 9: 157-180, 2017.

19. Molife LR, Yan L, Vitfell-Rasmussen J, Zernhelt AM, Sullivan DM, Cassier PA, Chen E, Biondo A, Tetteh E, Siu LL, et al: Phase 1 trial of the oral AKT inhibitor MK-2206 plus carboplatin/paclitaxel, docetaxel, or erlotinib in patients with advanced solid tumors. J Hematol Oncol 7: 1, 2014

20. Lara PN Jr,Longmate J, Mack PC, Kelly K, Socinski MA, Salgia R, Gitlitz B, Li T, Koczywas M, Reckamp KL and Gandara DR: Phase II study of the AKT inhibitor MK-2206 plus erlotinib in patients with advanced non-small cell lung cancer who previously progressed on erlotinib. Clin Cancer Res 21: 4321-4326, 2015

21. Ramanathan RK, McDonough SL, Kennecke HF, Iqbal S, Baranda JC, Seery TE, Lim HJ, Hezel AF, Vaccaro GM and Blanke CD: Phase 2 study of MK-2206, an allosteric inhibitor of AKT, as second-line therapy for advanced gastric and gastroesophageal junction cancer: A SWOG cooperative group trial (S 1005). Cancer 121: 2193-2197, 2015. 